\title{
RERESHMENT Hyperprolactinaemia and antipsychotics
}

\author{
James C. Nelson, Patrick M. Bell \& Stephen T. Guy
}

James C. Nelson is a consultant child and adolescent psychiatrist in the Northern Health and Social Care Trust, Belfast. Patrick M.

Bell is an Honorary Professor of Medicine and consultant physician at the Regional Centre for Endocrinology and Diabetes, Royal Victoria Hospital, Belfast. Stephen T. Guy is lead mental health pharmacist at Belfast Health and Social Care Trust, Belfast. Correspondence $\mathrm{Dr}$ James C. Nelson, Beechcroft Unit, 110 Saintfield Road, Belfast BT8 6HD, UK. Email: james.nelson@ northerntrust.hscni.net

\section{SUMMARY \\ This overview explains antipsychotic-induced hyperprolactinaemia and offers guidance on the management of this common and underestimated problem in general psychiatric practice. \\ DECLARATION OF INTEREST \\ S.T.G. has participated in an advisory board for Roche Pharmaceuticals}

Prolactin is a polypeptide hormone secreted by the lactotroph cells of the anterior pituitary gland. It is responsible primarily for breast enlargement in pregnancy and subsequent milk production. Dopamine is the primary inhibitor of prolactin release, and it is produced in the hypothalamus by the tuberoinfundibular neurons, then transported to the pituitary gland via the hypophyseal portal circulation. Dopamine receptor antagonists such as antipsychotics cause hyperprolactinaemia by reducing inhibition of prolactin secretion.

Since prolactin inhibits the release of gonadotrophin-releasing hormone, hyperprolactinaemia can indirectly lead to decreased oestrogen levels in women and decreased testosterone levels in men (Haddad 2004).

Macroprolactin is a prolactin immunoglobulin complex with lower biological activity than prolactin. Some laboratories do not routinely measure macroprolactin, but those that do do not always show its contribution to the total prolactin reading. Clinicians should therefore always check whether reported total prolactin includes a macroprolactin component, especially in patients with asymptomatic hyperprolactinaemia. This can avoid unnecessary further investigation (Melmed 2011).

Prolactin is secreted in a pulsatile manner, typically peaking in the middle of the night and reaching a trough level after midday. The physiological peak can be four times greater than the trough (Haddad 2004). The normal range of serum prolactin is generally below $530 \mathrm{mIU} / \mathrm{L}$ $(25 \mathrm{ng} / \mathrm{mL})$ in women and $424 \mathrm{mIU} / \mathrm{L}(20 \mathrm{ng} / \mathrm{mL})$ in men (Peveler 2008).

\section{Symptoms of hyperprolactinaemia}

The primary symptoms of hyperprolactinaemia are galactorrhoea, menstrual disturbance, infertility, decreased libido and gynaecomastia. Psychiatrists may fail to detect these symptoms either because they do not ask about them directly, or because patients are embarrassed to mention them or do not think them a medication side-effect (Haddad 2004).

\section{Causes of hyperprolactinaemia}

Physiological causes of hyperprolactinaemia include pregnancy, lactation, stress, sexual intercourse and sleep. There are a large number of pathological causes, including prolactinoma (usually associated with extremely elevated prolactin levels), chronic renal failure, liver cirrhosis, trauma and seizures. Non-functioning pituitary tumours can cause pituitary stalk compression and mild hyperprolactinaemia. Importantly, therefore, mild hyperprolactinaemia does not rule out a pituitary mass lesion (Melmed 2011).

Many medications can raise prolactin, particularly antipsychotics, but also antidepressants, oral oestrogens, oral contraceptives and opioids. Hyperprolactinaemia is particularly associated with amisulpride, risperidone and first-generation antipsychotics (Table 1). Nearly all antidepressants are associated with hyperprolactinaemia, though to a much lesser extent than antipsychotics. Clomipramine is the antidepressant most associated

\section{TABLE 1 Effects of antipsychotics on prolactin levels}

\begin{tabular}{|lc|}
\hline Antipsychotic & Increase in prolactin \\
\hline Amisulpride & +++ \\
\hline Risperidone & +++ \\
\hline First-generation antipsychotics & +++ \\
\hline Olanzapine & + \\
\hline Ouetiapine & + \\
\hline Clozapine & 0 \\
\hline Aripiprazole & 0 \\
\hline
\end{tabular}

0 , no effect; + , increase to abnormal levels in small percentage of patients; ++ , increase to abnormal levels in $25-50 \%$ of patients; +++ , increase to abnormal levels in $>50 \%$ of patients.

Adapted from Molitch (2008) and Holt \& Peveler (2011). 
with hyperprolactinaemia (Molitch 2008). Routine monitoring of prolactin when prescribing antidepressants is not recommended (Coker 2010).

\section{Long-term consequences of hyperprolactinaemia}

Hyperprolactinaemia is associated with loss of bone mineral density as a consequence of sex hormone deficiency (Haddad 2004). Even a few months of mild hyperprolactinaemia with amenorrhoea may affect bone mineral density (Peveler 2008).

Data suggest that prolactin has an important role in the development of some breast cancers (Tworoger 2007, 2013) and prolactin elevation should be avoided where there is a history of breast cancer (Peveler 2008).

\section{Clinical management of patients on antipsychotics}

Psychiatrists should inform patients about the symptoms of hyperprolactinaemia and enquire about these periodically. There is no consensus in various clinical guidelines about routine measurement of serum prolactin. Obtaining a baseline prolactin reading is strongly advised when commencing an antipsychotic associated with hyperprolactinaemia. Additionally, it appears prudent to measure serum prolactin 3 months after dose stabilisation and 3 months after any dose increase (Peveler 2008).

In the presence of a recent normal baseline prolactin level, hyperprolactinaemia following initiation of an antipsychotic is almost certainly medication induced. If confirmation of this is required, clinicians might consider discontinuing an oral antipsychotic as a trial for 3 days, by which time a drug-induced hyperprolactinaemia will usually normalise (Melmed 2011); this approach might not be feasible because of the risk of relapse.

The following management strategies may also be considered:

- review the need for ongoing antipsychotic medication; if appropriate, the antipsychotic might be discontinued permanently

- reduce the dose of antipsychotic (Haddad 2004)

- change the antipsychotic to one with a lower risk of hyperprolactinaemia (Melmed 2011).

If hyperprolactinaemia above $1000 \mathrm{mIU} / \mathrm{L}$ persists and aetiology is unclear, it may be necessary to discuss with an endocrinologist and consider other investigations, including liver, renal and thyroid function tests. In some cases, magnetic resonance imaging (MRI) of the hypothalamus and pituitary to exclude a mass lesion might be considered. A history of headaches or visual field disturbance raises the suspicion of a pituitary tumour.
When it is necessary to continue the antipsychotic in the presence of hyperprolactinaemia, the following could be considered, after consultation with an endocrinologist:

- adding oestrogen or testosterone for patients with long-term hypogonadism (hypogonadal symptoms or low bone mass) associated with hyperprolactinaemia (Melmed 2011)

- bone mineral density measurement for women with hyperprolactinaemia and 12 months of amenorrhoea (Haddad 2004)

- adding aripiprazole as an adjunct to the existing antipsychotic, which may normalise antipsychoticinduced hyperprolactinaemia (Shim 2007)

- adding a dopamine agonist such as bromocriptine or cabergoline, as this can normalise the prolactin level; this strategy carries a risk of exacerbating a psychosis (Chang 2008; Molitch 2008).

\section{Conclusions}

Antipsychotic-induced hyperprolactinaemia is a common and overlooked problem with potential long-term consequences. This overview offers some guidance on management, but difficult clinical judgements may still be required. Careful prolactin monitoring, including a baseline result for all patients receiving prolactin-raising antipsychotics, should be considered just as important as prelithium renal and thyroid function tests.

\section{References}

Chang SC, Chen C-H, Lu M-L (2008) Cabergoline-induced psychotic exacerbation in schizophrenic patients. General Hospital Psychiatry, 30: 378-80.

Coker F, Taylor D (2010) Antidepressant-induced hyperprolactinaemia incidence, mechanisms and management. CNS Drugs, 24: 563-74.

Haddad PM, Wieck A (2004) Antipsychotic-induced hyperprolactinaemia mechanisms, clinical features and management. Drugs, 64: 2291-314.

Holt RIG, Peveler RC (2011) Antipsychotics and hyperprolactinaemia: mechanisms, consequences and management. Clinical Endocrinology, 74: 141-7.

Melmed S, Casanueva FF, Hoffman AR, et al (2011) Diagnosis and treatment of hyperprolactinemia: an Endocrine Society clinical practice guideline. Journal of Clinical Endocrinology \& Metabolism, 96: 273-88.

Molitch ME (2008) Drugs and prolactin. Pituitary, 11: 209-18.

Peveler RC, Branford D, Citrome L, et al (2008) Antipsychotics and hyperprolactinaemia: clinical recommendations. Journal of Psychopharmacology, 22 (suppl 2): s98-103.

Shim JC, Shin JG, Kelly DL, et al (2007) Adjunctive treatment with a dopamine partial agonist, aripiprazole, for antipsychotic-induced hyperprolactinemia: a placebo-controlled trial. American Journal of Psychiatry, 164: $1404-10$.

Tworoger SS, Eliassen AH, Sluss P, et al (2007) A prospective study of plasma prolactin concentrations and risk of premenopausal and postmenopausal breast cancer. Journal of Clinical Oncology, 25: 1482-8.

Tworoger SS, Eliassen AH, Zhang X, et al (2013) A 20-year prospective study of plasma prolactin as a risk marker of breast cancer development. Cancer Research, 73: 4810-9. 